\title{
Inclusion results for certain subclasses of p-valent meromorphic functions associated with a new operator
}

\section{AO Mostafa*}

\section{"Correspondence:}

adelaeg254@yahoo.com

Department of Mathematics,

Faculty of Science, Mansoura

University, Mansoura, 35516, Egypt

\begin{abstract}
In this paper, we introduce new subclasses of $p$-valent starlike, $p$-valent convex, $p$-valent close-to-convex, and p-valent quasi-convex meromorphic functions and investigate some inclusion properties of these subclasses and investigate various inclusion properties and integral-preserving properties for the $\mathrm{p}$-valent meromorphic function classes.
\end{abstract}

MSC: $30 C 45$

Keywords: $p$-valent meromorphic functions; Hadamard product; inclusion properties

\section{Introduction}

Let $\Sigma_{p}$ denote the class of functions of the form

$$
f(z)=z^{-p}+\sum_{k=1}^{\infty} a_{k-p} z^{k-p} \quad(p \in \mathbb{N}=\{1,2, \ldots\})
$$

which are analytic and p-valent in the punctured unit disc $U^{*}=\{z: z \in \mathbb{C}$ and $0<|z|<1\}$. If $f(z)$ and $g(z)$ are analytic in $U=U^{*} \cup\{0\}$, we say that $f(z)$ is subordinate to $g(z)$, written $f \prec g$ or $f(z) \prec g(z)(z \in U)$, if there exists a Schwarz function $w(z)$ in $U$ with $w(0)=0$ and $|w(z)|<1$, such that $f(z)=g(w(z))(z \in U)$. Furthermore, if $g(z)$ is univalent in $U$, then the following equivalence relationship holds true (see [3] and [8]):

$$
f(z) \prec g(z) \quad \Leftrightarrow \quad f(0)=g(0) \text { and } f(U) \subset g(U) .
$$

For functions $f(z) \in \Sigma_{p}$, given by (1.1) and $g(z) \in \Sigma_{p}$ defined by

$$
g(z)=z^{-p}+\sum_{k=1}^{\infty} b_{k-p} z^{k-p} \quad(p \in \mathbb{N}),
$$

the Hadamard product (or convolution) of $f(z)$ and $g(z)$ is given by

$$
(f * g)(z)=z^{-p}+\sum_{k=1}^{\infty} a_{k-p} b_{k-p} z^{k-p}=(g * f)(z) .
$$

\section{Springer}

( 2012 Mostafa; licensee Springer. This is an Open Access article distributed under the terms of the Creative Commons Attribution License (http://creativecommons.org/licenses/by/2.0), which permits unrestricted use, distribution, and reproduction in any medium, provided the original work is properly cited. 
Aqlan et al. [1] defined the operator $Q_{\beta, p}^{\alpha}: \Sigma_{p} \rightarrow \Sigma_{p}$ by:

$$
Q_{\beta, p}^{\alpha} f(z)= \begin{cases}z^{-p}+\frac{\Gamma(\alpha+\beta)}{\Gamma(\beta)} \sum_{k=1}^{\infty} \frac{\Gamma(k+\beta)}{\Gamma(k+\beta+\alpha)} a_{k-p} z^{k-p} & \left(\alpha>0 ; \beta>-1 ; p \in \mathbb{N} ; f \in \Sigma_{p}\right), \\ f(z) & \left(\alpha=0 ; \beta>-1 ; p \in \mathbb{N} ; f \in \Sigma_{p}\right) .\end{cases}
$$

Now, we define the operator $H_{p, \beta, \mu}^{\alpha}: \Sigma_{p} \rightarrow \Sigma_{p}$ as follows:

First, put

$$
G_{\beta, p}^{\alpha}(z)=z^{-p}+\frac{\Gamma(\alpha+\beta)}{\Gamma(\beta)} \sum_{k=1}^{\infty} \frac{\Gamma(k+\beta)}{\Gamma(k+\beta+\alpha)} z^{k-p} \quad(p \in \mathbb{N})
$$

and let $G_{\beta, p, \mu}^{\alpha *}$ be defined by

$$
G_{\beta, p}^{\alpha}(z) * G_{\beta, p, \mu}^{\alpha *}(z)=\frac{1}{z^{p}(1-z)^{\mu}} \quad(\mu>0 ; p \in \mathbb{N}) .
$$

Then

$$
H_{p, \beta, \mu}^{\alpha} f(z)=G_{\beta, p}^{\alpha *}(z) * f(z) \quad\left(f \in \Sigma_{p}\right)
$$

Using (1.5)-(1.7), we have

$$
H_{p, \beta, \mu}^{\alpha} f(z)=z^{-p}+\frac{\Gamma(\beta)}{\Gamma(\alpha+\beta)} \sum_{k=1}^{\infty} \frac{\Gamma(k+\beta+\alpha)(\mu)_{k}}{\Gamma(k+\beta)(1)_{k}} a_{k-p} z^{k-p}
$$

where $f \in \Sigma_{p}$ is in the form (1.1) and $(\nu)_{n}$ denotes the Pochhammer symbol given by

$$
(v)_{n}=\frac{\Gamma(v+n)}{\Gamma(v)}= \begin{cases}1 & (n=0), \\ v(v+1) \cdots(v+n-1) & (n \in \mathbb{N}) .\end{cases}
$$

It is readily verified from (1.8) that

$$
z\left(H_{p, \beta, \mu}^{\alpha} f(z)\right)^{\prime}=(\alpha+\beta) H_{p, \beta, \mu}^{\alpha+1} f(z)-(\alpha+\beta+p) H_{p, \beta, \mu}^{\alpha} f(z)
$$

and

$$
z\left(H_{p, \beta, \mu}^{\alpha} f(z)\right)^{\prime}=\mu H_{p, \beta, \mu+1}^{\alpha} f(z)-(\mu+p) H_{p, \beta, \mu}^{\alpha} f(z)
$$

It is noticed that, putting $\mu=1$ in (1.8), we obtain the operator

$$
H_{p, \beta, 1}^{\alpha} f(z)=z^{-p}+\frac{\Gamma(\beta)}{\Gamma(\alpha+\beta)} \sum_{k=1}^{\infty} \frac{\Gamma(k+\alpha+\beta)}{\Gamma(k+\beta)} a_{k-p} z^{k-p}
$$

Let $M$ be the class of analytic functions $h(z)$ with $h(0)=1$, which are convex and univalent in $U$ and satisfy $\operatorname{Re}\{h(z)\}>0(z \in U)$.

For $0 \leq \eta, \gamma<p$, we denote by $\Sigma_{p} S(\eta), \Sigma_{p} K(\eta), \Sigma_{p} C(\eta, \gamma)$, and $\Sigma_{p} C^{*}(\eta, \gamma)$, the subclasses of $\Sigma_{p}$ consisting of all p-valent meromorphic functions which are, respectively, 
starlike of order $\eta$, convex of order $\eta$, close-to-convex functions of order $\gamma$ and type $\eta$, and quasi-convex functions of order $\gamma$ and type $\eta$ in $U$.

Making use of the principle of subordination between analytic functions, we introduce the subclasses $\Sigma_{p} S(\eta ; \phi), \Sigma_{p} C(\eta ; \phi), \Sigma_{p} K(\eta, \gamma ; \phi, \psi)$, and $\Sigma_{p} K^{*}(\eta, \gamma ; \phi, \psi)(0 \leq \eta, \gamma<p$ and $\phi, \psi \in M)$ of the class $\Sigma_{p}$ which are defined by:

$$
\begin{aligned}
& \Sigma_{p} S(\eta ; \phi)=\left\{f \in \Sigma_{p}: \frac{1}{p-\eta}\left(-\frac{z f^{\prime}(z)}{f(z)}-\eta\right) \prec \phi(z), \text { in } U\right\}, \\
& \Sigma_{p} K(\eta ; \phi)=\left\{f \in \Sigma_{p}: \frac{1}{p-\eta}\left(-\left[1+\frac{z f^{\prime \prime}(z)}{f^{\prime}(z)}\right]-\eta\right) \prec \phi(z), \text { in } U\right\}, \\
& \Sigma_{p} C(\eta, \gamma ; \phi, \psi)=\left\{f \in \Sigma_{p}: \exists g \in \Sigma_{p} S(\eta ; \phi) \text { s.t. } \frac{1}{p-\gamma}\left(-\frac{z f^{\prime}(z)}{g(z)}-\gamma\right) \prec \psi(z), \text { in } U\right\},
\end{aligned}
$$

and

$$
\begin{aligned}
& \Sigma_{p} C^{*}(\eta, \gamma ; \phi, \psi) \\
& \quad=\left\{f \in \Sigma_{p}: \exists g \in \Sigma_{p} K(\eta ; \phi) \text { s.t. } \frac{1}{p-\gamma}\left(-\frac{\left(z f^{\prime}(z)\right)^{\prime}}{g^{\prime}(z)}-\gamma\right) \prec \psi(z), \text { in } U\right\} .
\end{aligned}
$$

From these definitions, we can obtain some well-known subclasses of $\Sigma_{p}$ by special choices of the functions $\phi$ and $\psi$ as well as special choices of $\eta, \gamma$, and $p$ (see $[2,5]$, and [10]).

Now, by using the linear operator $H_{p, \beta, \mu}^{\alpha}(\alpha \geq 0, \mu>0, \beta>-1 ; p \in \mathbb{N})$ and for $\phi, \psi \in M$, $0 \leq \eta, \gamma<p$, we define new subclasses of meromorphic functions of $\Sigma_{p}$ by:

$$
\begin{aligned}
& \Sigma_{p} S_{\beta, \mu}^{\alpha}(\eta ; \phi)=\left\{f \in \Sigma_{p}: H_{p, \beta, \mu}^{\alpha} f \in \Sigma_{p} S(\eta ; \phi)\right\}, \\
& \Sigma_{p} K_{\beta, \mu}^{\alpha}(\eta ; \phi)=\left\{f \in \Sigma_{p}: H_{p, \beta, \mu}^{\alpha} f \in \Sigma_{p} K(\eta ; \phi)\right\}, \\
& \Sigma_{p} C_{\beta, \mu}^{\alpha}(\eta, \gamma ; \phi, \psi)=\left\{f \in \Sigma_{p}: H_{p, \beta, \mu}^{\alpha} f \in \Sigma_{p} C(\eta, \gamma ; \phi, \psi)\right\},
\end{aligned}
$$

and

$$
\Sigma_{p} C_{\beta, \mu}^{\alpha *}(\eta, \gamma ; \phi, \psi)=\left\{f \in \Sigma_{p}: H_{p, \beta, \mu}^{\alpha} f \in \Sigma_{p} C^{*}(\eta, \gamma ; \phi, \psi)\right\} .
$$

We also note that

$$
f(z) \in \Sigma_{p} K_{\beta, \mu}^{\alpha}(\eta ; \phi) \quad \Leftrightarrow \quad-\frac{z f^{\prime}(z)}{p} \in \Sigma_{p} S_{\beta, \mu}^{\alpha}(\eta ; \phi)
$$

and

$$
f(z) \in \Sigma_{p} C_{\beta, \mu}^{\alpha *}(\eta, \gamma ; \phi, \psi) \Leftrightarrow \quad-\frac{z f^{\prime}(z)}{p} \in \Sigma_{p} C_{\beta, \mu}^{\alpha}(\eta, \gamma ; \phi, \psi) .
$$

In particular, we set

$$
\Sigma_{p} S_{\beta, \mu}^{\alpha}\left(\eta ; \frac{1+A z}{1+B z}\right)=\Sigma_{p} S_{\beta, \mu}^{\alpha}(\eta ; A, B) \quad(-1<B<A \leq 1)
$$


and

$$
\Sigma_{p} K_{\beta, \mu}^{\alpha}\left(\eta ; \frac{1+A z}{1+B z}\right)=\Sigma_{p} K_{\beta, \mu}^{\alpha}(\eta ; A, B) \quad(-1<B<A \leq 1) .
$$

In this paper, we investigate several inclusion properties of the classes $\Sigma_{p} S_{\beta, \mu}^{\alpha}(\eta ; \phi)$, $\Sigma_{p} K_{\beta, \mu}^{\alpha}(\eta ; \phi), \quad \Sigma_{p} C_{\beta, \mu}^{\alpha}(\eta, \gamma ; \phi, \psi)$, and $\Sigma_{p} C_{\beta, \mu}^{\alpha *}(\eta, \gamma ; \phi, \psi)$ associated with the operator $H_{p, \beta, \mu}^{\alpha}$. Some applications involving integral operators are also considered.

In order to establish our main results, we need the following lemmas.

Lemma 1 [4] Let $\varsigma$ and $v$ be complex constants and let $h(z)$ be convex (univalent) in $U$ with $h(0)=1$ and $\operatorname{Re}\{\varsigma h(z)+v\}>0$. If

$$
q(z)=1+q_{1} z+\cdots
$$

is analytic in $U$, then

$$
q(z)+\frac{z q^{\prime}(z)}{\varsigma q(z)+v} \prec h(z) \quad(z \in U)
$$

implies

$$
q(z) \prec h(z) \quad(z \in U) .
$$

Lemma 2 [7] Let $h(z)$ be convex (univalent) in $U$ and $\psi(z)$ be analytic in $U$ with $\operatorname{Re}\{\psi(z)\} \geq 0$. If $q$ is analytic in $U$ and $q(0)=h(0)$, then

$$
q(z)+\psi(z) z q^{\prime}(z) \prec h(z) \quad(z \in U)
$$

implies

$$
q(z) \prec h(z) \quad(z \in U) .
$$

\section{Some inclusion results}

In this section, we give some inclusion properties for meromorphic function classes, which are associated with the operator $H_{p, \beta, \mu}^{\alpha}$. Unless otherwise mentioned, we assume that $\alpha \geq$ $1, \beta>-1, \mu>0,0 \leq \gamma, \eta<p$ and $p \in \mathbb{N}$.

Theorem 1 For $f(z) \in \Sigma_{p}, \phi \in M$ with

$$
\max _{z \in U}(\operatorname{Re}\{\phi(z)\})<\min _{z \in U}\left[\frac{p+\mu-\eta}{p-\eta}, \frac{\alpha+\beta+p-\eta}{p-\eta}\right],
$$

then we have

$$
\Sigma_{p} S_{\beta, \mu+1}^{\alpha}(\eta, \phi) \subset \Sigma_{p} S_{\beta, \mu}^{\alpha}(\eta, \phi) \subset \Sigma_{p} S_{\beta, \mu}^{\alpha-1}(\eta, \phi) .
$$


Proof We will first show that

$$
\Sigma_{p} S_{\beta, \mu+1}^{\alpha}(\eta, \phi) \subset \Sigma_{p} S_{\beta, \mu}^{\alpha}(\eta, \phi)
$$

Let $f \in \Sigma_{p} S_{\beta, \mu+1}^{\alpha}(\eta ; \phi)$ and put

$$
q(z)=\frac{1}{p-\eta}\left(-\frac{z\left(H_{p, \beta, \mu}^{\alpha} f(z)\right)^{\prime}}{H_{p, \beta, \mu}^{\alpha} f(z)}-\eta\right)
$$

where $q(z)$ is analytic in $U$ with $q(0)=1$. Applying (1.10) in (2.3), we have

$$
-\mu \frac{H_{p, \beta, \mu+1}^{\alpha} f(z)}{H_{p, \beta, \mu}^{\alpha} f(z)}=(p-\eta) q(z)+\eta-(p+\mu) .
$$

Differentiating (2.4) logarithmically with respect to $z$, we have

$$
\frac{1}{p-\eta}\left(-\frac{z\left(H_{p, \beta, \mu+1}^{\alpha} f(z)\right)^{\prime}}{H_{p, \beta, \mu+1}^{\alpha} f(z)}-\eta\right)=q(z)+\frac{z q^{\prime}(z)}{(p+\mu)-\eta-(p-\eta) q(z)} \quad(z \in U) .
$$

Since

$$
\max _{z \in U}(\operatorname{Re}\{\phi(z)\})<\min _{z \in U} \frac{p+\mu-\eta}{p-\eta}
$$

we see that

$$
\operatorname{Re}\{(p+\mu)-\eta-(p-\eta) \phi(z)\}>0 \quad(z \in U) .
$$

Applying Lemma 1 to (2.5), it follows that $q \prec \phi$, that is, that $f \in \Sigma_{p} S_{\beta, \mu}^{\alpha}(\eta ; \phi)$. The proof of the second part will follow by using arguments similar to those used in the first part with $f \in \Sigma_{p} S_{\beta, \mu}^{\alpha}(\eta ; \phi)$ and using the identity (1.9) instead of (1.10). This completes the proof of Theorem 1 .

Theorem 2 For $f(z) \in \Sigma_{p}, \phi \in M$ with

$$
\begin{aligned}
& \max _{z \in U}(\operatorname{Re}\{\phi(z)\})<\min _{z \in U}\left[\frac{p+\mu-\eta}{p-\eta}, \frac{\alpha+\beta+p-\eta}{p-\eta}\right], \\
& \Sigma_{p} K_{\beta, \mu+1}^{\alpha}(\eta ; \phi) \subset \Sigma_{p} K_{\beta, \mu}^{\alpha}(\eta ; \phi) \subset \Sigma_{p} K_{\beta, \mu}^{\alpha-1}(\eta ; \phi) \quad(0 \leq \eta<p ; \phi \in M) .
\end{aligned}
$$

Proof Applying (1.10) and using Theorem 1, we have

$$
\begin{aligned}
f(z) \in \Sigma_{p} K_{\beta, \mu+1}^{\alpha}(\eta ; \phi) & \Leftrightarrow \quad H_{p, \beta, \mu+1}^{\alpha} f(z) \in \Sigma_{p} K(\eta ; \phi) \\
& \Leftrightarrow \quad-\frac{z\left(H_{p, \beta, \mu+1}^{\alpha} f(z)\right)^{\prime}}{p} \in \Sigma_{p} S(\eta ; \phi) \\
& \Leftrightarrow \quad H_{p, \beta, \mu+1}^{\alpha}\left(-\frac{z f^{\prime}(z)}{p}\right) \in \Sigma_{p} S(\eta ; \phi) \\
& \Leftrightarrow \quad-\frac{z f^{\prime}(z)}{p} \in \Sigma_{p} S_{\beta, \mu+1}^{\alpha}(\eta ; \phi)
\end{aligned}
$$




$$
\begin{aligned}
& \Rightarrow \quad-\frac{z f^{\prime}(z)}{p} \in \Sigma_{p} S_{\beta, \mu}^{\alpha}(\eta ; \phi) \\
& \Leftrightarrow \quad H_{p, \beta, \mu}^{\alpha}\left(-\frac{z f^{\prime}(z)}{p}\right) \in \Sigma_{p} S(\eta ; \phi) \\
& \Leftrightarrow \quad-\frac{z\left(H_{p, \beta, \mu}^{\alpha} f(z)\right)^{\prime}}{p} \in \Sigma_{p} S(\eta ; \phi) \\
& \Leftrightarrow \quad H_{p, \beta, \mu}^{\alpha} f(z) \in \Sigma_{p} K(\eta ; \phi) \\
& \Leftrightarrow \quad f(z) \in \Sigma_{p} K_{\beta, \mu}^{\alpha}(\eta ; \phi) .
\end{aligned}
$$

Also,

$$
\begin{aligned}
f(z) \in \Sigma_{p} K_{\beta, \mu}^{\alpha}(\eta ; \phi) & \Leftrightarrow \quad-\frac{z f^{\prime}(z)}{p} \in \Sigma_{p} S_{\beta, \mu}^{\alpha}(\eta ; \phi) \\
& \Rightarrow \quad-\frac{z f^{\prime}(z)}{p} \in \Sigma_{p} S_{\beta, \mu}^{\alpha-1}(\eta ; \phi) \\
& \Leftrightarrow \quad-\frac{z\left(H_{p, \beta, \mu}^{\alpha-1} f(z)\right)^{\prime}}{p} \in \Sigma_{p} S(\eta ; \phi) \\
& \Leftrightarrow \quad H_{p, \beta, \mu}^{\alpha-1} f(z) \in \Sigma_{p} K(\eta ; \phi) \\
& \Leftrightarrow f(z) \in \Sigma_{p} K_{\beta, \mu}^{\alpha-1}(\eta ; \phi) .
\end{aligned}
$$

This completes the proof of Theorem 2 .

Taking

$$
\phi(z)=\frac{1+A z}{1+B z} \quad(-1<B<A \leq 1 ; z \in U)
$$

in Theorem 1 and Theorem 2, we have the following corollary.

Corollary 1 Let $f(z) \in \Sigma_{p}$ and

$$
\frac{1+A}{1+B}<\min _{z \in U}\left(\frac{p+\mu-\eta}{p-\eta}, \frac{\alpha+\beta+p-\eta}{p-\eta}\right) \quad(-1<B<A \leq 1) .
$$

Then we have

$$
\Sigma_{p} S_{\beta, \mu+1}^{\alpha}(\eta ; A, B) \subset \Sigma_{p} S_{\beta, \mu}^{\alpha}(\eta ; A, B) \subset \Sigma_{p} S_{\beta, \mu}^{\alpha-1}(\eta ; A, B)
$$

and

$$
\Sigma_{p} K_{\beta, \mu+1}^{\alpha}(\eta ; A, B) \subset \Sigma_{p} K_{\beta, \mu}^{\alpha}(\eta ; A, B) \subset \Sigma_{p} K_{\beta, \mu}^{\alpha-1}(\eta ; A, B)
$$

Now, using Lemma 2, we obtain similar inclusion relations for the subclass $\Sigma_{p} C_{\beta, \mu}^{\alpha}(\eta$; $\gamma ; \phi, \psi)$.

Theorem 3 Let $f(z) \in \Sigma_{p}$ and

$$
\max _{z \in U} \operatorname{Re}\{\phi(z)\}<\min _{z \in U}\left(\frac{p+\mu-\eta}{p-\eta}, \frac{\alpha+\beta+p-\eta}{p-\eta}\right) .
$$


Then we have

$$
\begin{aligned}
& \Sigma_{p} C_{\beta, \mu+1}^{\alpha}(\eta ; \gamma ; \phi, \psi) \subset \Sigma_{p} C_{\beta, \mu}^{\alpha}(\eta ; \gamma ; \phi, \psi) \subset \Sigma_{p} C_{\beta, \mu}^{\alpha-1}(\eta ; \gamma ; \phi, \psi) \\
& \quad(0 \leq \eta, \gamma<p ; \phi, \psi \in M) .
\end{aligned}
$$

Proof First, we will prove that

$$
\Sigma_{p} C_{\beta, \mu+1}^{\alpha}(\eta ; \gamma ; \phi, \psi) \subset \Sigma_{p} C_{\beta, \mu}^{\alpha}(\eta ; \gamma ; \phi, \psi)
$$

Let $f \in \Sigma_{p} C_{\beta, \mu+1}^{\alpha}(\eta ; \gamma ; \phi, \psi)$. Then, from the definition of the class $\Sigma_{p} C_{\beta, \mu}^{\alpha}(\eta ; \gamma ; \phi, \psi)$, there exists a function $g \in \Sigma_{p} S_{\beta, \mu}^{\alpha}(\eta ; \phi)$ such that

$$
\frac{1}{p-\gamma}\left(-\frac{z\left(H_{p, \beta, \mu+1}^{\alpha} f(z)\right)^{\prime}}{H_{p, \beta, \mu+1}^{\alpha} g(z)}-\gamma\right) \prec \psi(z) \quad(z \in U) .
$$

Now, let

$$
q(z)=\frac{1}{p-\gamma}\left(-\frac{z\left(H_{p, \beta, \mu}^{\alpha} f(z)\right)^{\prime}}{H_{p, \beta, \mu}^{\alpha} g(z)}-\gamma\right),
$$

where $q(z)$ is analytic in $U$ with $q(0)=1$. Applying (1.10) in (2.6), we have

$$
\begin{aligned}
& \frac{1}{p-\gamma}\left(-\frac{z\left(H_{p, \beta, \mu+1}^{\alpha} f(z)\right)^{\prime}}{H_{p, \beta, \mu+1}^{\alpha} g(z)}-\gamma\right) \\
& =\frac{1}{p-\gamma}\left(\frac{H_{p, \beta, \mu+1}^{\alpha}\left(\frac{-z f^{\prime}(z)}{p}\right)}{H_{p, \beta, \mu+1}^{\alpha} g(z)}-\gamma\right) \\
& =\frac{1}{p-\gamma}\left(\frac{z\left(H_{p, \beta, \mu}^{\alpha}\left(-\frac{z f^{\prime}(z)}{p}\right)\right)^{\prime}+(p+\mu) H_{p, \beta, \mu}^{\alpha}\left(-\frac{z f^{\prime}(z)}{p}\right)}{z\left(H_{p, \beta, \mu}^{\alpha} g(z)\right)^{\prime}+(p+\mu) H_{p, \beta, \mu}^{\alpha} g(z)}-\gamma\right) \\
& =\frac{1}{p-\gamma}\left(\frac{\frac{z\left(H_{p, \beta, \mu}^{\alpha}\left(-\frac{z f^{\prime}(z)}{p}\right)\right)^{\prime}}{H_{p, \beta, \mu}^{\alpha} g(z)}+(p+\mu) \frac{H_{p, \beta, \mu}^{\alpha}\left(-\frac{z f^{\prime}(z)}{p}\right)}{H_{p, \beta, \mu}^{\alpha} g(z)}}{\frac{z\left(H_{p, \beta, \mu}^{\alpha} g(z)\right)^{\prime}}{H_{p, \beta, \mu}^{\alpha} g(z)}+(p+\mu)}-\gamma\right) .
\end{aligned}
$$

Since, by Theorem 1 ,

$$
g(z) \in \Sigma_{p} S_{\beta, \mu+1}^{\alpha}(\eta ; \phi) \subset \Sigma_{p} S_{\beta, \mu}^{\alpha}(\eta ; \phi)
$$

set

$$
h(z)=\frac{1}{p-\eta}\left(-\frac{z\left(H_{p, \beta, \mu}^{\alpha} g(z)\right)^{\prime}}{H_{p, \beta, \mu}^{\alpha} g(z)}-\eta\right)
$$

where $h \prec \phi$ in $U$, and $\phi \in M$. Then, using (2.7) and (2.8), we have

$$
H_{p, \beta, \mu}^{\alpha}\left(-\frac{z f^{\prime}(z)}{p}\right)=[(p-\gamma) q(z)+\gamma] H_{p, \beta, \mu}^{\alpha} g(z)
$$


and

$$
\begin{aligned}
& \frac{1}{p-\gamma}\left(-\frac{z\left(H_{p, \beta, \mu+1}^{\alpha} f(z)\right)^{\prime}}{H_{p, \beta, \mu+1}^{\alpha} g(z)}-\gamma\right) \\
& =\frac{1}{p-\gamma}\left(\frac{\frac{z\left(H_{p, \beta, \mu}^{\alpha}\left(-\frac{z f^{\prime}(z)}{p}\right)\right)^{\prime}}{H_{p, \beta, \mu}^{\alpha} g(z)}+(p+\mu)[(p-\gamma) q(z)+\gamma]}{p+\mu-\eta-(p-\eta) h(z)}-\gamma\right) .
\end{aligned}
$$

Differentiating both sides of (2.9) with respect to $z$ and multiplying by $z$, we have

$$
\frac{z\left(H_{p, \beta, \mu}^{\alpha}\left(-\frac{z f^{\prime}(z)}{p}\right)\right)^{\prime}}{H_{p, \beta, \mu}^{\alpha} g(z)}=(p-\gamma) z q^{\prime}(z)-[(p-\gamma) q(z)+\gamma][(p-\eta) h(z)+\eta] .
$$

Making use of (2.6), (2.10), and (2.11), we have

$$
\frac{1}{p-\gamma}\left(-\frac{z\left(H_{p, \beta, \mu+1}^{\alpha} f(z)\right)^{\prime}}{H_{p, \beta, \mu+1}^{\alpha} g(z)}-\gamma\right)=q(z)+\frac{z q^{\prime}(z)}{p+\mu-\eta-(p-\eta) h(z)} \prec \psi(z), \quad z \in U
$$

Since $h \prec \phi$ in U, and

$$
\max _{z \in U} \operatorname{Re}\{h(z)\}<\frac{p+\mu-\eta}{p-\eta}
$$

then

$$
\operatorname{Re}\{p+\mu-\eta-(p-\eta) h(z)\}>0 \quad(z \in U) .
$$

Hence, putting

$$
\chi(z)=\frac{1}{\{p+\mu-\eta-(p-\eta) h(z)\}}
$$

in Eq. (2.12) and applying Lemma 2, we can show that $q \prec \psi$, that is, that $f \in \Sigma_{p} C_{\beta, \mu}^{\alpha}(\eta$, $\gamma ; \phi, \psi)$. The second part can be proved by using similar arguments and using (1.9). This completes the proof of Theorem 3 .

\section{Inclusion properties involving the integral operator $F_{p, \delta}$}

Now, we consider the generalized Libera integral operator $F_{p, \delta}(f)$ (see [6] and [9]), defined by

$$
F_{p, \delta}(f)(z)=\frac{\delta}{z^{\delta+p}} \int_{0}^{z} t^{\delta+p-1} f(t) d t=z^{-p}+\sum_{k=1}^{\infty} \frac{\delta}{\delta+k} a_{k-p} z^{k-p} \quad(\delta>-p) .
$$

From (3.1), we have

$$
z\left(H_{p, \beta, \mu}^{\sigma} F_{p, \delta}(f)(z)\right)^{\prime}=\delta H_{p, \beta, \mu}^{\sigma} f(z)-(\delta+p) H_{p, \beta, \mu}^{\sigma} F_{p, \delta}(f)(z) .
$$


Theorem 4 Let $\phi \in M$ with

$$
\max _{z \in U}(\operatorname{Re}\{\phi(z)\})<\frac{\delta+p-\eta}{p-\eta} \quad(\delta>-p) .
$$

Iff $\in \Sigma_{p} S_{\beta, \mu}^{\alpha}(\eta ; \phi)$, then $F_{p, \delta}(f) \in \Sigma_{p} S_{\beta, \mu}^{\alpha}(\eta ; \phi)$.

Proof Let $f \in \Sigma_{p} S_{\beta, \mu}^{\alpha}(\eta ; \phi)$ and put

$$
h(z)=\frac{1}{p-\eta}\left(-\frac{z\left(H_{p, \beta, \mu}^{\sigma} F_{p, \delta}(f)(z)\right)^{\prime}}{H_{p, \beta, \mu}^{\sigma} F_{p, \delta}(f)(z)}-\eta\right),
$$

where $h$ is analytic in $U$ with $h(0)=1$. Then, by using (3.2) and (3.3), we have

$$
-\delta \frac{H_{p, \beta, \mu}^{\sigma} f(z)}{H_{p, \beta, \mu}^{\sigma} F_{p, \delta}(f)(z)}=(p-\eta) h(z)+\eta-(p+\delta) .
$$

Differentiating (3.4) logarithmically with respect to $z$, we have

$$
\frac{1}{p-\eta}\left(-\frac{z\left(H_{p, \beta, \mu}^{\sigma} f(z)\right)^{\prime}}{H_{p, \beta, \mu}^{\sigma} f(z)}-\eta\right)=h(z)+\frac{z h^{\prime}(z)}{p+\delta-\eta-(p-\eta) h(z)} \quad(z \in U) .
$$

Applying Lemma 1, we conclude that $h \prec \phi(z \in U)$, which implies that $F_{p, \delta}(f) \in$ $\Sigma_{p} S_{\beta, \mu}^{\alpha}(\eta ; \phi)$.

Theorem 5 Let $\phi \in M$ with

$$
\max _{z \in U}(\operatorname{Re}\{\phi(z)\})<\frac{\delta+p-\eta}{p-\eta} \quad(\delta>-p) .
$$

If $\in \Sigma_{p} K_{\beta, \mu}^{\alpha}(\eta ; \phi)$, then $F_{p, \delta}(f) \in \Sigma_{p} K_{\beta, \mu}^{\alpha}(\eta ; \phi)$.

Proof Applying Theorem 4 and (1.12), we have

$$
\begin{aligned}
f(z) \in \Sigma_{p} K_{\beta, \mu}^{\alpha}(\eta ; \phi) & \Leftrightarrow \quad-\frac{z f^{\prime}(z)}{p} \in \Sigma_{p} S_{\beta, \mu}^{\alpha}(\eta ; \phi) \\
& \Rightarrow \quad F_{p, \delta}\left(-\frac{z f^{\prime}(z)}{p}\right)(z) \in \Sigma_{p} S_{\beta, \mu}^{\alpha}(\eta ; \phi) \\
& \Leftrightarrow \quad-\frac{z}{p} F_{p, \delta}^{\prime}(f)(z) \in \Sigma_{p} S_{\beta, \mu}^{\alpha}(\eta ; \phi) \\
& \Leftrightarrow \quad F_{p, \delta}(f)(z) \in K_{p, \lambda}^{\sigma}(\eta ; \phi) .
\end{aligned}
$$

This completes the proof of Theorem 5 .

From Theorem 4 and Theorem 5, we have the following corollary.

Corollary 2 Suppose that

$$
\frac{1+A}{1+B}<\frac{\delta+p-\eta}{p-\eta} \quad(\delta>-p ;-1<B<A \leq 1) .
$$


Then, for the classes $\Sigma_{p} S_{\beta, \mu}^{\alpha}(\eta ; \phi)$ and $\Sigma_{p} K_{\beta, \mu}^{\alpha}(\eta ; \phi)$, the following inclusion relations hold true:

$$
f \in \Sigma_{p} S_{\beta, \mu}^{\alpha}(A, B) \quad \Rightarrow \quad F_{p, \delta}(f) \in \Sigma_{p} S_{\beta, \mu}^{\alpha}(A, B)
$$

and

$$
f \in \Sigma_{p} K_{\beta, \mu}^{\alpha}(A, B) \quad \Rightarrow \quad F_{p, \delta}(f) \in \Sigma_{p} K_{\beta, \mu}^{\alpha}(A, B) .
$$

Theorem 6 Let $\phi, \psi \in M$ with

$$
\max _{z \in U} \operatorname{Re}\{\phi(z)\}<\frac{\delta+p-\eta}{p-\eta} \quad(\delta>-p)
$$

Iff $\in \Sigma_{p} C_{\beta, \mu}^{\alpha}(\eta, \gamma ; \phi, \psi)$, then $F_{p, \delta}(f) \in \Sigma_{p} C_{\beta, \mu}^{\alpha}(\eta, \gamma ; \phi, \psi)$.

Proof Let $f \in \Sigma_{p} C_{\beta, \mu}^{\alpha}(\eta, \gamma ; \phi, \psi)$. Then, from the definition of the class $\Sigma_{p} C_{\beta, \mu}^{\alpha}(\eta, \gamma ; \phi, \psi)$, there exists a function $g \in \Sigma_{p} S_{\beta, \mu}^{\alpha}(\eta ; \phi)$ such that

$$
\frac{1}{p-\gamma}\left(-\frac{z\left(H_{p, \beta, \mu}^{\sigma} f(z)\right)^{\prime}}{H_{p, \beta, \mu}^{\sigma} g(z)}-\gamma\right) \prec \psi(z) \quad(z \in U) .
$$

Now, let

$$
h(z)=\frac{1}{p-\gamma}\left(-\frac{z\left(H_{p, \beta, \mu}^{\sigma} F_{p, \delta}(f)(z)\right)^{\prime}}{H_{p, \beta, \mu}^{\sigma} F_{p, \delta}(g)(z)}-\gamma\right),
$$

where $h(z)$ is analytic in $U$ with $h(0)=1$. Applying (3.2) in (3.6), we have

$$
\begin{aligned}
& \frac{1}{p-\gamma}\left(-\frac{z\left(H_{p, \beta, \mu}^{\sigma} f(z)\right)^{\prime}}{H_{p, \beta, \mu}^{\sigma} g(z)}-\gamma\right) \\
& =\frac{1}{p-\gamma}\left(\frac{\left(H_{p, \beta, \mu}^{\sigma}\left(-\frac{z f^{\prime}(z)}{p}\right)(z)\right)^{\prime}}{H_{p, \beta, \mu}^{\sigma} g(z)}-\gamma\right) \\
& =\frac{1}{p-\gamma}\left\{\frac{z\left(H_{p, \beta, \mu}^{\sigma} F_{p, \delta}\left(-\frac{z f^{\prime}(z)}{p}\right)(z)\right)^{\prime}+(p+\delta) H_{p, \beta, \mu}^{\sigma} F_{p, \delta}\left(-\frac{z f^{\prime}(z)}{p}\right)}{z\left(H_{p, \beta, \mu}^{\sigma} F_{p, \delta}(g)(z)\right)^{\prime}+(p+\delta) H_{p, \beta, \mu}^{\sigma} F_{p, \delta}(g)(z)}-\gamma\right\} \\
& =\frac{1}{p-\gamma}\left\{\frac{\frac{z\left(H_{p, \beta, \mu}^{\sigma} F_{p, \delta}\left(-\frac{z f^{\prime}(z)}{p}\right)(z)\right)^{\prime}}{H_{p, \beta, \mu}^{\sigma} F_{p, \delta}(g)(z)}+(p+\delta) \frac{H_{p, \beta, \mu}^{\sigma} F_{p, \delta}\left(-\frac{z f^{\prime}(z)}{p}\right)}{H_{p, \beta, \mu}^{\sigma} F_{p, \delta}(g)(z)}}{\frac{z\left(H_{p, \beta, \mu}^{\sigma} F_{p, \delta}(g)(z)\right)^{\prime}}{H_{p, \beta, \mu}^{\sigma} F_{p, \delta}(g)(z)}+p+\delta}-\gamma\right\} .
\end{aligned}
$$

Since $g \in \Sigma_{p} S_{\beta, \mu}^{\alpha}(\eta ; \phi)$, then by Theorem 4 , we have $F_{p, \delta}(g)(z) \in \Sigma_{p} S_{\beta, \mu}^{\alpha}(\eta ; \phi)$. Let

$$
q(z)=\frac{1}{p-\eta}\left(-\frac{z\left(H_{p, \beta, \mu}^{\sigma} F_{p, \delta}(g)(z)\right)^{\prime}}{H_{p, \beta, \mu}^{\sigma} F_{p, \delta} g(z)}-\eta\right),
$$


where $q \prec \phi$ in $U$. Then, using the same techniques as in the proof of Theorem 3 and using (3.5) and (3.7), we have

$$
\frac{1}{p-\gamma}\left(-\frac{z\left(H_{p, \beta, \mu}^{\sigma} f(z)\right)^{\prime}}{H_{p, \beta, \mu}^{\sigma} g(z)}-\gamma\right)=h(z)+\frac{z h^{\prime}(z)}{\delta+p-\eta-(p-\eta) q(z)} \prec \psi(z)
$$

Since $\operatorname{Re}\left\{\frac{1}{\delta+p-\eta-(p-\eta) q(z)}\right\}>0$, then applying Lemma 2, we find that $h \prec \psi$, which yields $F_{p, \delta}(f)(z) \in \Sigma_{p} C_{\beta, \mu}^{\alpha}(\eta, \gamma ; \phi, \psi)$. This completes the proof of Theorem 6.

Remark Putting $\mu=1$ in the above results, we obtain the results corresponding to the operator $H_{p, \beta, 1}^{\alpha}$ defined by (1.11).

\section{Competing interests}

The author declares that she has no competing interests.

\section{Author's contributions}

The author read and approved the .nal manuscript.

Received: 21 May 2012 Accepted: 18 July 2012 Published: 31 July 2012

\section{References}

1. Aglan, E, Jahangiri, JM, Kulkarni, SR: Certain integral operators applied to meromorphic $p$-valent functions. J. Nat. Geom. 24, 111-120 (2003)

2. Bajpai, SK: A note on a class of meromorphic univalent functions. Rev. Roum. Math. Pures Appl. 22, $295-297$ (1977)

3. Bulboaca, T: Differential Subordinations and Superordinations. Recent Results. House of Scientific Book Publ., Cluj-Napoca, 2005

4. Eenigenburg, P, Miller, SS, Mocanu, PT, Reade, MO: On a Briot-Bouquet differential subordination. In: General Inequalities 3. Internat. Schriftenreihe Numer. Math., vol. 64, pp. 339-348. Birkhäuser, Basel (1983)

5. Goel, RM, Sohi, NS: On a class of meromorphic functions. Glasnik Math. III, 17(37), 19-28 (1982)

6. Libera, RJ: Some classes of regular univalent functions. Proc. Am. Math. Soc. 16, 755-758 (1965)

7. Miller, SS, Mocanu, PT: Differential subordinations and univalent functions. Mich. Math. J. 28(2), 157-171 (1981)

8. Miller, SS, Mocanu, PT: Differential Subordination: Theory and Applications. Series on Monographs and Textbooks in Pure and Applied Mathematics, vol. 225. Dekker, New York (2000)

9. Owa, S, Srivastava, HM: Some applications of the generalized Libera integral operator. Proc. Jpn. Acad., Ser. A, Math. Sci. 62, 125-128 (1986)

10. Singh, R: Meromorphic close-to-convex functions. J. Indian Math. Soc. 33, 13-20 (1969)

doi:10.1186/1029-242X-2012-169

Cite this article as: Mostafa: Inclusion results for certain subclasses of p-valent meromorphic functions associated with a new operator. Journal of Inequalities and Applications 2012 2012:169.

\section{Submit your manuscript to a SpringerOpen ${ }^{\circ}$ journal and benefit from:}

- Convenient online submission

- Rigorous peer review

- Immediate publication on acceptance

- Open access: articles freely available online

- High visibility within the field

- Retaining the copyright to your article 